\title{
Rievocando Guido Davide Neri
}

La morte di Guido Davide Neri, di cui quest'anno ricorre il ventennale, ha determinato la presa di coscienza - nei suoi maestri, amici, colleghi e allievi - dell'irreparabilità dell'evento, della definitività del venir meno di una voce così teoreticamente stimolante ed eclettica. Perdita tragicamente vissuta nella cerchia della sua frequentazione, ma, parallelamente, inserita nell'ampio contesto del dibattito culturale nazionale e internazionale, in cui Neri era organicamente inserito. Pare a noi, che non lo abbiamo conosciuto e gli siamo temporalmente più distanti rispetto a quanti ne hanno sinora scritto, quasi inusuale che, a un filosofo mancato da così poco tempo, sia stata consacrata una già così importante quantità di ricerche e studi. Al di là della retorica che spesso accompagna, in ambiente accademico, il ritiro dall'attività didattica o la scomparsa di un docente, e abituati alla necessità della sedimentazione storica per la messa a punto del bilancio storiografico, ci ha stupito l'intensità con cui Neri è stato ricordato e la varietà degli interventi sulla sua figura, l'insegnamento e la produzione scientifica.

Se da un lato questo acuisce, in noi, il rimpianto di non averlo incrociato nel mondo, dall'altro stimola la volontà di tornare a lui, al suo pensiero, alle sue opere. Un ritorno che, non mediato dall'influenza personale, potrà certo apparire privo di taluni elementi importanti, come la testimonianza diretta $o$ la compartecipazione a certe esperienze determinanti, ma, insieme, si potrà giovare del giusto distacco critico, trovando ora nel prezioso Fondo Guido Davide Neri, conservato presso l'Università degli Studi di Milano, la fonte privilegiata dell'accesso alla sua opera teorica. Questo, si potrebbe dire, è il fil rouge che accomuna i nostri brevi contributi, diversi per tematiche e occasione di elaborazione, ma tutti contraddistinti dallinteresse e dalla volontà di avvicinare un po' meglio la figura di Neri: le carte dell'archivio 
personale per noi hanno rappresentato e rappresentano la possibilità di accedere al suo laboratorio, di scoprire da esse l'uomo e il filosofo e di dare concretezza documentaria alla dialettica tra pensiero, letture ed esperienze.

Certo il lavoro da svolgere è ancora molto e solo lo scandaglio approfondito del materiale permetterà di penetrare a fondo l'officina neriana. Quello che noi qui, più limitatamente, offriamo, è soltanto un piccolo esempio di quanto di rilevante e ancora da vedere è racchiuso nei faldoni e aspetta di essere portato alla luce. Il nostro, in altre parole, vorrebbe essere un invito a ripensare Neri attraverso le carte, convinti della loro imprescindibilità ai fini della ricostruzione storico-critica del suo percorso filosofico e coscienti del loro straordinario valore intrinseco (per certi versi anche estetico). Gli interventi che presentiamo - ne siamo convinti - sono poi una ulteriore testimonianza della capacità di Neri e del suo pensiero di porsi in dialogo, anche a distanza, con una sempre nuova varietà di interlocutori.

Luca Natali

Paolo Karapedian

Sofia Elena Merli 


\section{L'arte che difende e «si difende»}

\section{di Luca Natali}

luca.natali@unimi.it

Che Guido Davide Neri possedesse una profonda conoscenza del tessuto urbano, della storia e delle chiese della sua Milano è quasi superfluo ricordarlo; in lui l'interesse e la passione per i fenomeni artistici e il radicamento nel mondo-della-vita del quartiere e della città suoi natali erano fortemente saldati: «amava questa città e sosteneva che occorressero gesti simbolici verso le sue chiese, l'architettura delle sue case, per riparare l'insensatezza dilagante, che ne faceva qualcosa di altro» 1 .

Ed è a partire dall'analisi fenomenologica, estetica e storico-artistica di una delle più significative incarnazioni milanesi dell'arte rinascimentale lombarda, da lui amata, che prende le mosse l'inedito neriano che qui si presenta. Ancor più interessante e significativo in quanto incentrato su quell'interfacciarsi dei due atteggiamenti metafisici - uno chiuso e statico, l'altro aperto e dinamico - che hanno come correlato i due mondi, del pensiero libero e della prassi vitale da una parte e delle risposte precostituite e della quotidianità banalizzante del lavoro dall'altra. Il «perdere di significato della propria esistenza», per usare un'espressione husserliana cara a Neri, conduce la società (europea), «mediante il prevalere delle "scienze di fatto"», a «ridurre gli uomini a "uomini di fatto"»", che danno forma e vivono in città dei fatti, dal

\footnotetext{
${ }^{1}$ C. Zamboni, "Nota biografica", in G. D. Neri, Il sensibile, la storia, l'arte. Scritti 1957-2001, Ombre corte, Verona 2003, p. 357. Cfr. anche A. Vigorelli, Guido D. Neri, Rivista di storia della filosofia, LIX, 2004, p. 777.

${ }^{2}$ G. D. Neri, Prassi e conoscenza, Feltrinelli, Milano 1966, p. 14.
} 
tumultuoso andirivieni professionale, a cui idealmente Neri contrappone la lentezza e la profondità della città dei monumenti, dei musei, delle chiese ${ }^{3}$.

Siamo nei primi anni '90. Sono questi anni in cui gli interventi pubblici di Neri divengono piuttosto rari: tra il '91 e il '95 dà alle stampe un denso saggio husserliano, un breve contributo sull'«idea "filosofica" di Europa" e un omaggio al maestro Dino Formaggio"; bisognerà poi attendere il '97 perché questa sostanziale «afasia» si interrompa ${ }^{5}$. Non si deve però pensare sia, quello isolato, un periodo di stasi intellettuale. La ricca ed eclettica attività didattica, testimoniata dai programmi dei corsi tenuti a Verona ${ }^{6}$, nonché gli impegni come conferenziere tra Milano, Brescia, Verona e Trento ${ }^{7}$, sono lo specchio di una incessante ricerca scientifica e di un continuato dialogo e scambio teoretico. I suoi taccuini conservano inoltre le tracce del lavoro di ripensamento e approfondimento dei temi che saranno poi alla base degli

\footnotetext{
${ }^{3}$ Preziosa, anche per una contestualizzazione dell'inedito qui proposto, la testimonianza, sul progetto di Neri di scrivere un libro su Milano, di L. Marelli, "Un filosofo indecisionista a Milano", in Quando tra noi muore un filosofo, Viciguerra, Pizzighettone 2002, pp. 30-31: «Mi sono chiesto quale Milano sarebbe emersa da un libro di Guido. Cosa non comune tra i milanesi conosceva veramente l'architettura e la storia dei suoi monumenti, le sue chiese, i suoi musei, i suoi artisti che ha fatto conoscere anche a me. Amava questa Milano, la sentiva familiare, la gustava anche quando da fine critico, ne metteva in luce gli aspetti a suo parere negativi. Non credo di sbagliarmi se penso che questa Milano fosse divenuta per Guido una sorta di rifugio al riparo da un'altra Milano, a lui estranea e pur sempre la sua città. La definiva la città non-città, precipitata in un caotico e spigoloso vuoto dove sono i fatti a farla da padroni, una città di fatti per "uomini di fatto". Da "indecisionista" Guido si collocava ai bordi di questa città, il cui spirito decisionista vedeva incarnato, con spudorato orgoglio, in un tetro groviglio di vie e piazze senza sbocco. Quando, dopo averle attraversate, entrava in una chiesa a rivedere le opere dei suoi amati pittori lombardi si sentiva accogliere in uno spazio benevolo continuamente rinnovantesi, dove l'eco del passato si traduceva in un messaggio verso il futuro».

${ }^{4}$ G. D. Neri, Terra e cielo in un manoscritto husserliano del 1934, aut aut, CCXLV, 1991, pp. 19-44; G. D. Neri, "Intorno all'idea "filosofica" di Europa", in Azione e contemplazione. Scritti in onore di U. Pellegrino, IPL, Milano 1992, pp. 415-424; G. D. Neri, Dino Formaggio uomo d'arte, in Il canto di Seikilos. Scritti per Dino Formaggio nell'ottantesimo compleanno, Guerini e Associati, Milano 1995, pp. 131-133.

5 Riprendo il termine da M. Carbone, "Neri, Caravaggio e l'interrogazione del silenzio", Materiali di Estetica, XI, 2004, pp. 102, 104.

${ }^{6}$ Cfr. L. Fausti, Guido Davide Neri tra scepsi e storia. Un percorso filosofico, Unicopli, Milano 2010, pp. 175-182.

${ }^{7}$ Cfr. ancora Ivi, p. 113 e L. Fausti, "Un filosofo della pòlis", in Quando tra noi muore un filosofo, cit., p. 42.
} 
scritti più importanti della fase successiva ${ }^{8}$. Risale in particolare al $1992-$ anno di stesura di gran parte del quaderno che qui si prenderà in considerazione - il ritorno alla riflessione su di un pittore amato sin dalla gioventù: Caravaggio ${ }^{9}$. Sui di lui Neri si soffermerà specificamente - è vero nel 1997, pubblicando su «aut aut» una lunga nota in cui discuterà l'interpretazione dell'artista lombardo proposta da Marc Fumaroli, ma è la mostra, vista a Firenze, Michelangelo Merisi da Caravaggio - Come nascono $i$ capolavori, ad offrire al filosofo l'occasione di ripensare al valore dell'arte caravaggesca ${ }^{10} \mathrm{e}$ di assorbire poi questa operazione in un più esteso discorso circa la possibilità di ricondurre (o limitare) la dirompente significatività dell'espressione artistica nelle maglie della ricostruzione storiograficoideologica del milieu vissuto e dello Zeitgeist sperimentato. Se, accanto a Fumaroli, saranno le opposte letture di Bologna e Calvesi, a consentire a Neri di far oscillare il pendolo interpretativo tra il realismo della pennellata caravaggesca e la sua eccedenza trascendente, è significativo trovare, tra le pagine del quaderno, uno dei germi della riappropriazione di Caravaggio da parte di Neri.

Scrive egli infatti:

\section{Caravaggio}

Sentita la confercenza> di Mina) Gregori su Caravaggio e il sacro, che riconferma la tesi di Mraurizio` Calvesi.

Caravaggio sarebbe un pittore della Rif ^orma〉 cattolica di S. Carlo Bøorromeo» e di S. Filippo Neri, S. Ignazio (?) ecc.

\footnotetext{
8 Cfr. Fondo Guido Davide Neri, Serie 1, Cartelle 8-12, u.a. 78-125.

9 Sulla fascinazione di Neri per l'artista lombardo si possono vedere, tra le altre, le testimonianze di G. Scaramuzza e P. Gambazzi, in Quando tra noi muore un filosofo, cit., pp. 17-18 e 28-29. Apprendo da Amedeo Vigorelli che Neri possedeva il manifesto della storica Mostra del Caravaggio e dei caravaggeschi del 1951, da lui visitata quando era ragazzo.

10 Cfr. quanto riporta G. Messetti, "Caravaggio a Firenze", in Quando tra noi muore un filosofo, cit., p. 50 e il catalogo della mostra a cura di Mina Gregori [Firenze, Palazzo Pitti, dicembre 1991-marzo 1992; Roma, Palazzo Ruspoli, marzo-maggio 1992] Michelangelo Merisi da Caravaggio - Come nascono i capolavori, Electa, Milano 1991.
} 
Invece: posto lo shock certo avuto da C'aravaggio〉 a Milano, con la peste ecc., certo lui porta a Roma quua〉lc‘osa〉 che gli viene di là (e da Venezia‘) ): non però la piega dolente e sottomessa, mistica e macerantesi, dei pittori controriformisti! porta la freschezza di Moretto e Savoldo ecc. Contro il Rinascimento morente, in questo è davvero lui la Riforma italiana. Non tanto di chiesa! è un riformatoreribelle che agisce in proprio, per atti teatrali concentrati in immagini.

Mi ha commosso rivedere ingigantito dalla proiezione il S. Matteo (distrutto) di Berlino ${ }^{11}$.

Difficile non scorgere in nuce, tra queste poche righe, la trama teorica di Caravaggio e la scuola del silenzio ${ }^{12}$, di quella irriducibilità e inderivabilità dell'«evento [...] Caravaggio» dai prodromi tecnico-artistici e culturali da cui la sua esperienza pittorica proveniva. Non la somma di quanto aveva appreso dai modelli lombardo-veneti è l'arte di Caravaggio; certo i citati Moretto e Savoldo, ma anche Ambrogio Figino, i Campi di Cremona o Peterzano, ebbero un certo peso nell'evoluzione del suo linguaggio, la cui carica sconvolgente non vi può essere però appiattita ${ }^{13}$. Non la teologia della controriforma è la sua religiosità, la crisi dei fondamenti post-rinascimentale è stata da lui vissuta da «protagonista [e] interprete» con «tutto se stesso»: nella «storia di una vita che si racconta» - la sua - e nelle «invenzioni drammatiche» di cui è stato capace, si apre un mondo nuovo, «un'alternativa» a quel pensiero

\footnotetext{
${ }^{11}$ Fondo Guido Davide Neri, Serie 1, Cartella 9, u.a. 98, c. num. $26 r$.

12 G. D. Neri, "Caravaggio e la scuola del silenzio", aut aut, CCLXXX-CCLXXXI, 1997, pp. 139-156.

${ }^{13} \mathrm{Nel}$ 1957, in uno dei suoi primi lavori scientifici, Neri affrontava il problema, in seno alle estetiche neoidealista e marxista, della storicizzazione dell'opera d'arte e del giudizio estetico, con una sensibilità, però, differente. Egli ricercava allora per il giudizio di valore sull'opera d'arte, una origine diversa da quella prospettata da Lionello Venturi, che lo voleva riportato nell'iperuranio della coincidenza tra "concetto universale» e «intuizione della singola opera d'arte», o di quella, sostenuta da Longhi, che introduceva «la totale storicità delle opere d'arte», facendo tuttavia permanere, nell'atto critico, quella dimensione ultrastorica rappresentata dal «magico scoccare della scintilla intuitiva, la quale sola ha a che fare con l'essenza dell'arte». Neri concludeva auspicando una messa in discussione dei fondamenti dell'estetica idealistica sin dalle fondamenta e non, come molti marxisti, una mera apposizione preliminare degli elementi di struttura (socio-economici) per potersi liberare la "coscienza dottrinaria» e poi "servirsi tranquillamente dei tradizionali schemi interpretativi dell'idealismo". Cfr. G. Neri, "Storiografia dell'arte: note", Ragionamenti, vIII, 1957, pp. 195197, ora in G. D. Neri, Il sensibile, la storia, l'arte, cit., pp. 303-305.
} 
rassicurante, che riassorbe la crisi e che informerà la cultura filosoficoletteraria del Sei- Settecento ${ }^{14}$.

La dialettica immanente-trascendente $o$, forse più correttamente, ancora quella tra chiusura e apertura, è alla base di altre riflessioni sviluppate nel quaderno del '92. Si trovano sul foglio immediatamente precedente alle osservazioni su Caravaggio, e il pungolo pare essere fornito da un rito funebre:

Sabato mattina Ultimo saluto a P`adre` D‘avid` M`aria` Turoldo in S. Carlo.

Moltissima gente, ha parlato Martini: commentando 3 passi evangelici

- Pasqua in casa di quua〉lçunos: il migliore tra voi sia servo (a differcenza〉 dei potenti)

- perché mi hai abbandonato

- Cristo risorto ricompare e dice come io sono venuto a voi, così andate agli altri e a chi rimetterete sarà rimesso, a chi no, no.

- I religiosi non compaiono tanto alla nascita (piuttosto: donne, medici) quanto alla morte (dove sembrano insostituibili)

- Sempre stare $1 / 2$ in chiesa e $1 / 2$ fuori?

- dire che si frequentano le chiese per le pitture?

- a volte mi chiedo: ma cosa mi hanno fatto di male i preti e la chiesa cattolica? Ecco: la violenza dell'imposizione ovvia. Sulle cose del pensiero e del credere amministriamo noi! Sta così e basta, impara. (=Il Catechismo). Il costume dei cattolici è di avere da sempre abdicato su questo punto. Forse spaventati dall'Inquisizione?

E i pretacci di religione? ${ }^{15}$

La morte di questo protagonista della vita spirituale ambrosiana e promotore del rinnovamento conciliare, costringe Neri a fare i conti col suo

\footnotetext{
${ }^{14}$ Cfr. G. D. Neri, "Caravaggio e la scuola del silenzio", cit., pp. 154-156. Assai significativi gli avantesti del saggio conservati nell'archivio personale, preziosi anche per un inquadramento del riemergere dell'interesse teorico neriano su Caravaggio: cfr. per es. Fondo Guido Davide Neri, Serie 1, Cartelle 10-11, u.a. 101-2, 106, 118-119. Ma sarebbero da tenere presenti anche i documenti contenuti in Serie 3, Sottoserie 4, Cartelle 27-29, u.a. 12, 14-26, 39-40, 45, 48, 62, 67-68.

${ }^{15}$ Fondo Guido Davide Neri, Serie 1, Cartella 9, u.a. 98, c. num. 25r.
} 
rapporto con la Chiesa cattolica, abbandonata da ragazzo, e con le chiese,

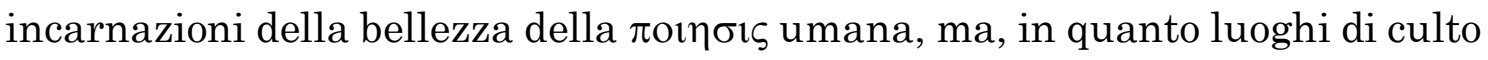
aperti a una dimensione ulteriore, non riducibili alla loro rappresentabilità estetica ${ }^{16}$. Ancora vi si nota la giustapposizione discordante tra un sapere passibile di critica e correzione e uno monolitico, in alcun modo permeabile alla domanda, al dubbio, alla sospensione di giudizio. È con questo spirito che Neri si ferma a scrutare la facciata di S. Pietro in Gessate, con uno sguardo che, alla luce delle annotazioni di cui si è detto e di altre che ora si vedranno, appare più studiato che occasionalmente determinato da un passaggio in Corso di Porta Vittoria ${ }^{17}$.

La tensione trascendente espressa dalla luce caravaggesca, ripresa e trasfigurata però dalla pittura della realtà lombarda, e l'incontro ultimo con le contrapposte religiosità viva e aperta al mondo di Padre Turoldo e arroccata nella fortezza delle verità di fede della Chiesa istituzionalizzata, deve aver esercitato un certo turbamento e generato, in Neri, la necessità di (ri)meditare su queste opposizioni in una forma che non parrebbe esaurirsi nella sfera privata, se quella da lui abbozzata ha le sembianze di una vera e propria 'lettera aperta':

$|51 r|$ Lettera sia a quelli che dicono di non credere che a quelli che ritengono di credere.

- St. Eustorgio vs. Palıazzo di Giustizia〉

- Chiese e conventi: non solo per guardare i quadri; l'architettura non è solo ... (Nomi)

\footnotetext{
${ }^{16} \mathrm{Su}$ Neri e il suo rapporto con le chiese e la Chiesa, illumina il racconto di R. Alquati in Quando tra noi muore un filosofo, cit., p. 20: «Malgrado non fossimo credenti parlavamo molto di religione e pure della chiesa oggi, e lui che da ragazzo realizzò la sua prima grande rottura e rivolta proprio contro la chiesa cattolica, adesso vedeva bene proprio certi aspetti del cattolicesimo. Ripeteva "ormai è solo sul sagrato delle chiese e nelle chiese al pomeriggio che si vedono persone riunite a parlare di certe grosse questioni della nostra vita"».

${ }_{17}$ Il lapsus contenuto nel testo manoscritto in riferimento a S. Eustorgio-S. Pietro in Gessate porta infatti ad avanzare l'ipotesi che la contrapposizione tra (una) chiesa e il Palazzo di Giustizia sia da ritenersi prima pensata e solo in un secondo momento descritta e analizzata dal vivo. Cfr. Fondo Guido Davide Neri, Serie 1, Cartella 9, u.a. 98, cc. num. 51r e 54r; in quest'ultima c. in particolare la lezione «S. P. in Gessate» corregge in linea «S. Eustorgio» cassata.
} 
- Chi li ha creati e gestiti - e ancora ci parlano - non può essermi estraneo.

In altro riguardo: chiese e conventi si intrecciano a una cultura dove il rapporto tra la vita e la morte è più forte e vero che "da noi". Il clero e ordini relig ciosi> hanno fatto la scelta di gestire la vita al suo estremo - la mantengono bene e male. Tagliati fuori dalla cultura moderna, anche loro hanno perso molto.

$|51 v|$ Da parte loro, hanno arrancato dietro la modernità, frenandola. Che Galileo sia "scopritore e copritore" non toglie che lui era vita e apertura, la Chiesa era morta.

Ma la cultura laica ha percorso il suo ciclo: cura della vita (del giorno) (Patıčka). (E gli effetti si vedono - fino al Golfo).

$|52 r|$

- conventi e pietas

- da S. Carlo a noi, i funerali

- laicismo moderno: storia

- i riti laici (Rivヶoluziones Frıancese» ecc.)

- architett<ura> ecclesiiastica〉 modkerna!!!18

Questo lo schema, molto asciutto, di un possibile intervento pubblico che è stato solo abbozzato e di cui resta però una redazione parziale tematicamente assai ricca.

Come si diceva, la contrapposizione, che poi emergerà nettamente nella parte concretamente stesa, è pensata prima che vissuta. D'altronde, non si tratta tanto, pur nella sua icasticità, dell'opposizione fisica, poi ripresa nel fronteggiarsi di S. Pietro in Gessate e del Palazzo di Giustizia, ma di quella «spiritual[e]» tra le diverse nature dei luoghi coinvolti. Da lì il discorso avrebbe poi dovuto allargarsi, investendo i simbolizzanti quanto i simbolizzati: la chiesa realtà visibile (e suo valore artistico) e la chiesa portatrice della carica di spiritualità infusale da chi l'ha costruita e «ancora ci parla[...]», attraverso l'eco di una cultura e di una fede che non può essere estranea e che pure fatica a sopravvivere nella modernità, anzi la combatte arrancando e avendo, a sua volta, perduto la propria vivacità a causa della scelta compiuta. Il Galileo pioniere del pensiero scientifico moderno e

${ }^{18}$ Fondo Guido Davide Neri, Serie 1, Cartella 9, u.a. 98, cc. num. 51r/v-52r. 
dell'ontologia ad esso associata, che Neri, sulle orme di Husserl ${ }^{19}$, aveva posto al vertice di quello scadimento della metafisica occidentale responsabile della riduzione della "razionalità a semplice "ratio" tecnico-utilitaria» 20 , è qui rivalutato in chiave positiva, rispetto alle forzature del dogma ecclesiastico. Certo, anche nell'inedito in questione emergerà poi una critica stringente (esibita anche iconograficamente) al riduzionismo tecnico-utilitaristico - da non confondere comunque con una inavvertita e irrazionale diffidenza nei confronti della scienza. Neri però sembra suggerire, a questo livello, che, se circostanziata nel preciso contesto storico, la novità galileiana era pur sempre «vita e apertura», costituiva la rottura di uno schema infrangibile di pensiero, una finestra di nuovo aperta sul mondo, mentre, dall'altra parte, la rigidità cattolica incarnava l'asfittica chiusura e, in definitiva, la morte dell'azione libera e creativa. Uno "slancio», quello della rivoluzione scientifica, che ha però «percorso il suo ciclo», scrive Neri, lasciando al non detto la valutazione sulle sue derive metafisiche e accostando alla prima annotazione una seconda, altrettanto breve ma densa, che evoca l'ethos patočkiano di una filosofia - cura dell'anima - obbligata al perenne rinnovarsi, avendo la sua peculiarità nella prospettiva e non nella dottrina. Il sotteso di queste righe sembra stare, per un verso, nella cognizione dell'impossibilità a liberarsi dalla tradizione e dal dogma attraverso la loro sostituzione con altre forme mitologiche (tecnico-scientifiche) e, per l'altro, nella posizione del potenziale valore emancipativo della costante apertura rappresentata dalla filosofia nel suo senso originario, mediato dallo $\theta \alpha \cup \mu \alpha \zeta \varepsilon ı v$, dallo shock, dallo stupore per

\footnotetext{
${ }^{19}$ Il riferimento è, naturalmente, al $\S 9$ della Crisi, da cui Neri trae anche l'espressione di Galileo quale «scopritore e copritore» («entdeckender und verdeckender»): cfr. E. Husserl, Die Krisis der europäischen Wissenschaften und die transzendentale Phänomenologie. Ein Einleitung in die phänomenologische Philosophie, hrsg. W. Biemel, «Husserliana», B. VI, Nijhoff, Haag 1954, p. 53; La crisi delle scienze europee e la fenomenologia trascendentale. Introduzione alla filosofia fenomenologica, tr. it. di E. Filippini, il Saggiatore, Milano 1961, p. 81 [edizione posseduta e annotata da Neri: cfr., nell'OPAC Unimi, 3L.GDN.T.HUS.03].

${ }^{20}$ G. D. Neri, "L'"ouverture" della Crisi", in E. Husserl, L'obiettivismo moderno. Riflessioni storico-critiche sul pensiero europeo dall'età di Galileo, a cura di G. D. Neri, Il Saggiatore, Milano 1976, pp. ix-liv, ora in Il sensibile, la storia, l'arte, cit., pp. 40-65, qui p. 57.
} 
il mondo che il sempre-nuovo-sguardo determina, in conflitto con la rassicurante narrazione della metafisica della quotidianità e della routine lavorativa ${ }^{21}$. L'esercizio della domanda è ciò che, sospendendo le facili e stereotipate risposte dei miti, consente di giungere alla libera pratica del pensiero, a quella necessità propria dell'uomo che sta nella ricerca, nel «bisogno di certezze». Non più, in questo caso, «soddisfatto da "risposte che precedono le domande" ${ }^{22}$, ma incalzato e continuamente problematizzato: ecco recuperata la dimensione autentica della storicità, della vita nella società, fondata sulla cura dell'anima che rinnega le comode e sedative spiegazioni dell'autorità (sia essa religiosa o politica), per aprirsi alla vertigine dell'interrogazione non irregimentata del mondo. Il passaggio, efficacemente esemplificato da Neri, è quello che porta dalla metafisica degli adulti-bambini a quella dei bambini-adulti23.

Di certo, come prospettata nel suo scheletro, questa "lettera" avrebbe, con ogni probabilità, toccato poi registri di discorso ancor più ampi; avrebbe indugiato sul significato della laicità, sulle diverse incarnazioni di questo termine («laicismo moderno: storia») e, probabilmente, riflettuto sulle contraddizioni di una cultura laica ormai completamente ripulita da ogni intrusione valoriale religiosa, le cui conseguenze, per la storia dell'umanità

${ }^{21}$ Per il soffermarsi di Neri su questi temi della filosofia di Patočka, vanno visti J. Patočka, Saggi eretici sulla filosofia della storia, CSEO, Bologna 1981; J. Patočka, Liberté et sacrifice. Écrits politiques, Millon, Grenoble 1990 e J. Patočka, Platon et l'Europe. Séminare privé du semestre d'été 1973, Verdier, Lagrasse 1983. Di quest'ultima opera esiste, nella biblioteca personale di Neri, una copia fittamente annotata, in cui l'emergere delle problematiche che saranno proprie del saggio "L'Europa dal fondo del suo declino" (1998) è reso palese dalle multiformi (e multicolori) sottolineature e dai marginalia. Cfr. p. 62 («le risposte precedono le domande»), p. 69 («il mito non ha stupore»), p. 99 («Europa = cura dell'anima vs cura del dominio») Cfr., nell'OPAC Unimi, 3L.GDN.T.PAT.01.

${ }_{22}^{2}$ G. D. Neri, "L'Europa dal fondo del suo declino", aut aut, CCLXXXIII-CCLXXXIV, 1998, p. 138. ${ }^{23}$ Cfr. G. D. Neri, "Metafisica dei bambini paragonata a quella degli adulti", C.R.I.F. Bollettino, XIII-XV, 1999, pp. 5-6, ora in Il sensibile, la storia, l'arte, cit., pp. 152-155. "I bambini sollevano la domanda, ma gli adulti non reggono il problema. Non sanno cosa dire perché non sanno cosa pensare, perché da tempo hanno scansato per se stessi le domande. Le hanno lasciate al prete oppure alla scienza, o a quelle "filosofie" totali che svolgono una funzione analoga. I bambini crescono e imparano a fare lo stesso, a non fare più domande. Così, da adulti, si comportano come i bambini, che cercano risposte rassicuranti dai genitori o dai maestri». Ivi, p. 153. 
occidentale, risultavano ben visibili nell'attualità allora più scottante («fino al Golfo») $)^{24}$.

Difficile raggiungere, attraverso la ricostruzione del testo progettato, tutti i rivoli teorici per i quali la meditazione di Neri avrebbe condotto questi contrasti; è preferibile, piuttosto, andare a quanto effettivamente realizzato, accettandone l'incompletezza insieme alla ricchezza concettuale e alla forte tensione dialogica.

Il testo principia dalla piccola piazza antistante S. Pietro in Gessate, nel tardo pomeriggio ("ore 17.45») di un giorno di fine inverno-inizio primavera ${ }^{25}$. Neri scrive mentre guarda la facciata del piccolo edificio, attorniato dai palazzi della Milano moderna; nota subito la sua capacità di ricavarsi uno spazio vitale all'interno della loro rigida geometricità, interrotta dallo slargo a ciottoli e dal doppio filare di piante. La descrizione storico-artistica della chiesa e la ricostruzione del suo possibile aspetto originario non è, in realtà, l'aspetto centrale dell'analisi di Neri. Non v'è dubbio che, oltre alle forme architettoniche, anche le condizioni di conservazione della chiesa influiscano sull'effetto visivo da essa prodotto; il «mattone fuligginoso», preso probabilmente come elemento di contrasto rispetto al biancastro/grigiastro dei palazzi circostanti e di quello di Giustizia, possiede un "valore di antico" termine preso in prestito da Alois Riegl ${ }^{26}$ - che «non guasta», tanto che il

${ }^{24}$ Sugli effetti di una certa laicità, connessa con la cultura del nichilismo e del consumo capitalistico, estrinsecantesi nella "pedagogia delle bombe», si veda, anche come possibile sviluppo del discorso schematizzato nel quaderno, G. D. Neri, "La guerra", in Il sensibile, la storia, l'arte, cit., pp. 292-296.

${ }_{25}$ Alcune annotazioni precedenti al testo qui preso in considerazione rimandano al febbraiomarzo 1992 (cfr. per es. Fondo Guido Davide Neri, Serie 1, Cartella 9, u.a. 98, cc. num. 29v, 37v.), mentre una successiva (Ivi, c. num. 60r) si riferisce alla futura Pasqua del '92 [19 aprile]: «Pasqua $=$ dove?».

${ }^{26}$ Cfr. per es. con A. Riegl, Der moderne Denkmalkultus. Sein Wesen und seine Entstehung, Braumüller, Wien und Leipzig 1903, p. 22, in cui si registra anche una possibile suggestione sulla capacità dell'Alterswert di agire a livello del pubblico non specialista (la "gente vecchia e stanca», infra): "Der Alterswert eines Denkmals verrät sich auf den ersten Blick durch dessen unmodernes Aussehen. Und zwar beruht dieses unmoderne Aussehen nicht so sehr auf der unmodernen Stilform, denn diese ließe sich ja auch imitieren, und ihre richtige Erkenntnis und Beurteilung wäre fast ausschließlich dem verhältnismäßig engen Kreise 
filosofo si «domand[a] con una certa apprensione se una pulitura troppo chiara non rovinerebbe l'effetto». Il discorso di Neri si sofferma, però, soprattutto sulla capacità di proteggersi esibita dalla costruzione, seppure «asserragliata», dai «blocchi quadrati» che «la sovrastavano». I mezzi di difesa sono, dice Neri, «spirituali»: la serie delle cappelle laterali - quelle della navata sinistra, scampate ai bombardamenti - attrae lo sguardo, obbligando l'occhio a rimanere a loro «aderente», «come se», viene specificato, la chiesa «volesse difendere me». Il carattere posseduto dal piccolo tempio è declinato anche in senso salvifico: la sua disomogeneità fenomenologico-estetica, rispetto al contesto, è potenzialità di riscatto da una modalità di vivere la città e il mondo determinata dallo sguardo dogmatico del quotidiano e incarnata nello stesso spazio architettonico (il Palazzo di Giustizia) ${ }^{27}$. La chiusura e l'apertura come coppia concettuale torna quindi all'interno dell'analisi estetica, per significare, ancora, il potere della vista che "ripensa" il mondo e ne rimane affascinato, in contrapposizione alla banalità dell'atteggiamento che tutto livella e mortifica nell'uso tecnico-pratico. Le "grosse panchine di granito», nella piazza e sotto gli alberi, offrono «riparo» e costituiscono, per la «gente vecchia e stanca», «un'isola» sulla quale sostare per estraniarsi dal «traffico» e dall'«andirivieni frettoloso del marciapiedi antistante», immagine di quel mondo degli affari che è l'espressione della razionalità operativa che fagocita le esistenze, ma non satura la profondità di senso di cui la piccola

gelernter Kunsthistoriker vorbehalten, während der Alterswert den Anspruch erhebt, auf die großen Massen zu wirken. Der Gegensatz zur Gegenwart, auf dem der Alterswert beruht, verrät sich vielmehr in einer Unvollkommenheit, einem Mangel an Geschlossenheit, einer Tendenz auf Auflösung der Form und Farbe, welche Eigenschaften denjenigen moderner, das heißt neuentstandener Gebilde schlankweg entgegengesetzt sind». Riegl è autore frequentato da Neri sin dagli anni della tesi di laurea (tit.: Teoria dell'arte e categorie storiografiche in Alois Riegl), discussa a Pavia sotto la guida di Formaggio e Paci, cfr. C. Zamboni, "Nota biografica", cit., p. 360 n. 5.

${ }_{27} \mathrm{Su}$ chiese e monumenti come "permanenze di cultura viva e luoghi di educazione dello spirito» cfr. quanto scrive L. Fausti, Guido Davide Neri tra scepsi e storia, cit., p. 25. 
chiesa si fa baluardo, offrendo un appiglio al quale aggrapparsi nel correre impetuoso della corrente ${ }^{28}$.

Questa polarità, arricchita da ulteriori, è trascinata, nelle righe che seguono, in un orizzonte discorsivo che trova la sua ispirazione allorché viene varcata la soglia. La specificità del luogo - in altre parole il suo significato trascendente - impone un certo atteggiamento nei confronti della stessa struttura fisica: che la porta sia «aperta» e che, perciò, Neri si consideri «un po' a casa", come nelle moschee da lui visitate in Algeria e Turchia, non toglie che il suo rapportarsi all'ambiente sia, in questo caso, assai più problematico. Alla radice v'è infatti una «rottura», quella con la religione "della [sua] famiglia». Una decisione drammatica e gravida di conseguenze, ancora peraltro agenti; lo conferma lo stesso filosofo, non esitando a definire la scelta un «trauma» e osservando come il recidere il legame col culto e la religione cattolica avesse determinato una sofferta lacerazione nei rapporti con «tanti parenti cari», incapaci di comprenderne le cause. Questo allontanamento, se proveniva da motivazioni già espresse riflettendo sul funerale di Padre Turoldo ed era stato la risposta all'avvertito carattere imposto e superstizioso di certe pratiche, agisce ora come sprone per considerare, sotto un'altra luce, dichiaratamente religiosa, il rapporto tra l'ambiente e le espressioni artistiche che in esso trovano luogo. Torna, sviluppato, il quesito sulla frequentazione delle chiese: si può dire che si «frequentano per le pitture?» $\mathrm{O}$ per la musica? Si può dire che il contesto percettivo sia neutro rispetto al valore estetico?

${ }^{28}$ Cfr. C. Dante in Quando tra noi muore un filosofo, cit., p. 55: «Guido mi ha mostrato un'altra Milano, quella che non hanno ancora bevuto [...]. La Milano delle chiese e dei luoghi meno conosciuti, la Milano degli anni Cinquanta [...]. Le volte che lo accompagnavo, molto tardi, alla stazione di Brescia, la Brescia deserta, guardava i grattacieli da poco spuntati e sottolineava le stridenti contraddizioni della città, tra passato e futuro, tra solidarietà ed economicismo, fra generosità e ruvidezza». 
Neri risponde di averlo creduto, di essere stato per lungo tempo sedotto dalla teoria della pura visibilit ${ }^{29}$, dalla presa in esame dell'opera indirizzata al rinvenimento dei modi formali e dei segni universali da essa presentati, a prescindere dai contenuti presenti. Il rappresentato gli appariva dunque «indifferente», svincolata com'era la realtà dell'opera d'arte da quella di qualsiasi dato naturale preesistente: che si trattasse di «Madonne e Santi» oppure di soggetti più 'laici', non faceva differenza per la valutazione estetica; come pure il dove: in Duomo o a Brera la "natura del soggetto», in quanto opera d'arte, non ne veniva alterata, il "soggetto vero" era comunque un altro» ${ }^{30}$.

Più sfaccettato è invece il giudizio presente nelle carte del quaderno: in certa misura al paradigma visibilista viene riconosciuta validità, parimenti però, si attribuisce una maggiore complessità al fenomeno estetico e al suo contesto di produzione ed esecuzione: l'ascolto della musica di Händel va considerato sempre ""pur[o]"»? In qualsiasi occasione si abbia modo di ascoltarla essa parla all'ascoltatore nello stesso identico modo? «In Chiesa come al Conservatorio?»: «In verità non era così». Con ogni evidenza il rapporto con la religione, tanto quella, affettivamente connotata, della cerchia famigliare, quanto quella, più distante e avversa, delle sue incarnazioni positive, rappresenta l'innesco anche per riconsiderare l'esperienza estetica dei monumenti religiosi, nell'ottica di una migliore messa a punto del suo

29 All'impostazione della reine Sichtbarkeit formulata da Konrad Fiedler (Über die Beurteilung von Werken der bildenden Kunst, Hirzel, Leipzig 1876), la cui eco italiana è rinvenibile in Benedetto Croce, si rifece anche Riegl, ancora evidente punto di riferimento delle osservazioni di Neri. Ancora a Riegl, e a Wölfflin, Neri faceva riferimento nella sua Introduzione a E. Panofsky, La prospettiva come "forma simbolica" e altri scritti, a cura di G. D. Neri, con una nota di M. Dalai, Feltrinelli, Milano 1961, pp. 7-33.

${ }^{30}$ In ogni caso Neri amava la pittura nordica, e fiamminga in particolare, anche perché, come racconta Alquati, è «rappresentazione di cose, mentre quella dell'Europa latina [è] rappresentazione di miti, soprattutto di quelli della Chiesa, e questo lo infastidiva. Citava un suo amico americano: «Sempre tutti questi santi e Madonne e basta!». «Qualche anno fa in una breve gita a Parma ad un certo punto rifiutò di guardare il Correggio ed anche il Parmigianino! Uscì imprecando dalla Steccata» (in Quando tra noi muore un filosofo, cit., p. 22). Cfr. infra. 
legame con «Chi li ha creati e gestiti», che, scrive ancora Neri, «non può essermi estraneo». Naturalmente la rivalutazione in atto ha come fulcro e punto di riferimento la religiosità nei suoi termini ideali e filosofici, eppure, di riflesso, le convenzioni sociali ad esse legate e, non ultimo, l'atteggiamento della gerarchia giocano un ruolo primario. Ad esempio la chiusura sperimentata nell'infanzia e nell'adolescenza, nella forma di un catechismo senza esitazioni o incertezze, viene qui messa in discussione, grazie al ricordo di alcune esperienze e testimonianze: l'eccezionalità di talune "figure di cattolici», il mutamento dell'indirizzo della Chiesa, l'«ammirazione» e la «meraviglia» per «le vicende di Solidarnosc» ${ }^{31}$, nonché l'inquietudine per una posizione politica, abbracciata in gioventù, che presentava tanti aspetti di consonanza con l'autoritarismo di setta ${ }^{32}$ e le cui ombre si allungavano «sinistr[e]» sul Novecento.

La fede, nella doppia accezione religiosa ${ }^{33}$ e politica: ecco il cuore delle riflessioni di Neri, del suo rimando a Galileo e alla ragione che imbriglia il

31 Sull'esperienza polacca si può vedere G. D. Neri, "Immagini del "dopo". Gli anni di Solidarnosc", L'ottavo giorno, 0, 1982, pp. 90-101, ora in Il sensibile, la storia, l'arte, cit., pp. 255-265. In questo saggio Neri utilizza, per descrivere la situazione in Cecoslovacchia e in Polonia dopo il fallimento del «movimento riformatore di Praga» e la «repressione preventiva degli studenti e degli intellettuali» nel contesto polacco, l'espressione «decadenza da tardo impero» (ivi, p. 255), ripresa anche nell'inedito («la triste atmosfera da tardo impero») per tratteggiare il clima post '68.

32 Diversi furono i contributi di Neri sul socialismo reale. Lasciando da parte il libro del 1980, forse il suo più importante, vale la pena di citare almeno: G. D. Neri, "L'esperienza cecoslovacca", Quaderni piacentini, XXXVI, 1968, pp. 11-46; G. D. Neri, "Variazioni ideologiche del socialismo realizzato: 1"umanismo" scientifico-tecnologico", aut aut, CXLV-CXLVI, 1975, pp. 51-72; G. D. Neri, "Sul significato del 1968-69 in Cecoslovacchia", aut aut, CXLVIII, 1975, pp. 39-59.

${ }^{3}$ A. Vigorelli ha parlato della presenza di un "'senso religioso"» in Neri, cfr. la presentazione a L. Fausti, Guido Davide Neri tra scepsi e storia, cit., p. 11. Una minuta di lettera di Neri a Renato Rozzi conferma la sua percezione della cogenza del problema religioso e della necessità per il mondo laico dell'apertura verso di esso. Nel contesto di un ripensamento dell'ora di religione nelle scuole italiane, il filosofo milanese scrive dell'opportunità di opporre alla "religione separata» dell'ortodossia cattolica, non "l'"alternativa alla religione"», ma «l'alternativa alla separatezza», offrendo "uno spazio di cultura e di problematica religiosa senza restrizioni» e discriminazioni: "Penso che l'attivazione di uno spazio problematico e aperto di cultura religiosa sarebbe un'innovazione seria per la cultura italiana, in quanto aprirebbe una buona competizione (anziché una cattiva dialettica) verso il monopolio cattolico della religione. Non la si potrebbe considerare come il frutto spontaneo della cultura laica moderna, ma sarebbe almeno un frutto maturo della sua capacità autocritica. Constatare che 
mondo nella dimensione tecnico-operativa, come anche del suo contrapporre le verità indiscusse al carattere invece fecondo della religione. Sebbene non utilizzi mai questo termine qui (se non al polo semantico opposto: «infedele»), è ad esso che, nella pagina, si allude. Egli giunge infatti sino a chiedersi, a fronte dei problemi che si è posto su religione, chiese, arte, musica, da dove derivi la "nuova sensibilità", il "nuovo sguardo" nella contemplazione di San Pietro in Gessate o delle note di Händel, non derubricabili a ""pura forma sensibile"»; rispondendosi, secondo un paradigma coerente con la sua impostazione teoretica, con una domanda: «Mi stavo convertendo? Rinnegavo tutto il mio passato e mi apprestavo a giocare il ruolo del figliol prodigo?».

Ripercorrendo brevemente il proprio cammino di formazione, il filosofo si sofferma sul passaggio dalla fede cattolica a quella politica, esponendo le ragioni di quella crisi religiosa, dalle radici anche culturali - gli echi carducciani di Versaglia, le letture di Kant, Feuerbach e Marx, il magistero banfiano -, che andava trovando consolazione nell'abbraccio con l'ideologia, giudicata, ex eventu, come un aspetto e non la radice della propria filosofia. La sua riflessione filosofica, le sue posizioni teoretiche non vengono poste in dubbio, nel loro valore e nel loro percorso; è, semmai, la genesi a venirne puntualizzata: «non rinuncio a un rigo della mia fillosofia», «[s]oltanto: non l'ho trovata nelle parti politiche cui mi ero avvicinato». Queste ultime

la provocazione viene in parte dall'esterno - da ciò che era stato escluso ma che continua a imporsi e dal fallimento di certezze storiche troppo caricate - significherebbe riconoscere che quella cultura non ha sempre convogliato in sé tutto il senso delle cose, che qualche volta lo ha anche travisato e che se le religioni storiche non sono l'ultima parola circa il nostro modo di essere al mondo, neppure l'ateismo come contrapposto al teismo, l'immanenza come opposta alla trascendenza o infine il naturalismo scientista come spiegazione totale hanno prodotto risposte definitive per la coscienza moderna. Forse il sentimento di questa duplice insufficienza [...] segnala che abbiamo bisogno di un nuovo punto di partenza». Assai rilevante anche il P.S. in calce alla lettera (da cfr. con infra): "A rinforzo delle ultime righe, che toccano problemi smisurati, propongo come tema di riflessione: ripensare il senso di immanenza e trascendenza alla luce della filosofia contemporanea, dopo Husserl, Heidegger, Bloch ecc.; oppure: verificare se quella vasta parte della cultura laica che si è formata per generazioni sul concetto seguente, espresso un secolo fa da G. Carduccci: / Decapitaro, Emmanuel Kant Iddio / Massimiliano Robespierre il re / può ancora considerarlo come una sintesi della propria autocoscienza storico-filosofica». Cfr. Fondo Guido Davide Neri, Serie 2, Cartella 16, u.a. 23, minuta datata «8 gennaio 1987». 
riproponevano infatti il catechismo «borioso e gretto» che lo avevano allontanato dalla Chiesa; piuttosto il marxismo "eterodosso" aveva rappresentato per Neri un referente di primo piano e un bacino teorico prezioso dal quale trarre spunti decisivi per la sua evoluzione, tanto teoretica quanto politica. Il soggiorno praghese dei primi anni '60, Prassi e conoscenza e il suo fare i conti con una impostazione del problema della praxis affrontando posizioni poco conosciute e poco canoniche, gli interventi sulla Primavera di Praga, ma, soprattutto Aporie della realizzazione, il libro che era stato un indicatore evidente di tale sensibilità. Non è possibile, in questa occasione, sezionare tutti i molteplici aspetti della filosofia della storia neriana, così come non è immaginabile riprendere le diverse e numerose fonti delle sue pagine. Un passo del quaderno suggerisce tuttavia un accostamento ad una metafora blochiana che si rivela evocativa della trama delle meditazioni imbastite da Neri a partire dalla visita alla piccola chiesa milanese.

Della scoperta del dissidente marxista tedesco, per lui così importante ${ }^{34}$, Neri scrive: «leggere Bloch era come ascoltare H«ä̊ndel o Bach. Si toccava il profondo». Agli occhi del filosofo milanese, se paragonati all'ambiente nel quale esercitava il suo pensiero, i temi di Bloch risultavano marcatamente inusuali (la religione e la morte, per Neri strettamente legate) ${ }^{35}$, ma a distinguersi, all'interno del suo côté, era soprattutto la modalità di ricerca, l'impostazione filosofica. Sul sostare e permanere in Neri di figure blochiane (Elena e Pamina), specialmente significative per illustrare il rapporto tra immagine utopica e realizzazione, è già stato sottolineato il rilievo ${ }^{36}$; qui

\footnotetext{
${ }^{34} \mathrm{Nel}$ 1979, con Laura Boella, Neri aveva curato un numero speciale di «aut aut» (il CLXXIIICLXXIV) dedicato all'Eredità di Bloch, il cui pensiero sarebbe stato un punto di riferimento essenziale all'interno della monografia dell'anno successivo Aporie della realizzazione. Anche a livello didattico Bloch era molto presente in quel periodo, in particolare si vedano i corsi del quadriennio 1977-1981 (cfr. L. Fausti, Guido Davide Neri tra scepsi e storia, cit., pp. 162-165). 35 Cfr. F. Papi, "Una vita filosofica", Materiali di Estetica, XI, 2004, p. 39.

${ }^{36}$ Cfr. L. Boella in Quando tra noi muore un filosofo, cit., p. 25; L. Fausti, Guido Davide Neri tra scepsi e storia, cit., pp. 69-72.
} 
invece traspare in filigrana più la coppia metaforica di Maria e Marta, così come è contenuta in Aporie. Là Neri, facendo emergere il tentativo blochiano di tenere insieme, nella riflessione sulla realizzazione socialista, «i due momenti della tensione escatologica e della fruizione» ${ }^{37}$, aveva rivendicato il valore dell'esortazione a non sacrificare, sull'altare della fattività tecnicopratica, la contemplazione:

\begin{abstract}
Viene in mente [...] l'invito rivolto da Bloch a un marxismo troppo incline a privilegiare il momento attivo su quello contemplativo - "Marta" rispetto a "Maria" nella simbologia del Principio Speranza - a saper riconoscere "quel momento di autenticità che addita all'attività il suo centro di quiete". Nel pensiero di Bloch, nonostante la sua intensa partecipazione per la "bruciante escatologia" del subito di Gesù e di Thomas Münzer, la realtà umana, la prassi autentica che le corrisponde, non ammette una contrapposizione tra attività $\mathrm{e}$ contemplazione, come pure - in un altro contesto - tra il momento arcadico e quello della costruzione, tra il pastore e l'homo faber; non ha quindi un carattere unilateralmente prometeico. Anzi proprio il momento arcadico, colto nella sua autenticità, assume "la funzione di un correttivo nei confronti di un'utopia sociale che troppo pianifica e surriscalda, di quella che è realmente giunta a costruire" e che si è resa responsabile della "perdita dello scopo nella costruzione del cammino"38.
\end{abstract}

$\mathrm{Su}$ queste stesse fondamenta si regge l'impalcatura dell'inedito: determinismo e libertà, superficialità di una vita attiva privata del "profondo" della contemplazione, genialità artistica e riduzione alle sue premesse storiche. La "città dei fatti", quella dei parallelepipedi e della febbrile alacrità, contro l'«asserragliata» chiesetta, presidio del bello, dello spirituale, del contemplativo; di un'arte che è salvifica e che costringe («mi tiene aderente») a soffermarsi su qualcosa che è altro rispetto al suo 'intorno': come la filosofia di Bloch, come le tele di Caravaggio. La dimensione nuova che crea l'evento dell'apertura sul mondo, senza preconcetti e senza finalità allotrie, come anche l'incontro con questa, costituisce il riparo, l'àncora di salvezza dall'autorità politica e religiosa, dal regime del pensiero unico, massificante $\mathrm{e}$ degradante, e rappresenta lo scarto emancipativo grazie al quale ritrovare

${ }^{37}$ G. D. Neri, Aporie della realizzazione. Filosofia e ideologia nel socialismo reale, Feltrinelli, Milano 1980, p. 77.

${ }^{38}$ Ivi, p. 78.

Materiali di Estetica - N. 8.2: 2021, Pagina 485 
l'umanità e il suo senso, mai dato per sempre, ma sempre di nuovo creato e cercato.

Si è detto della tendenza all’indecisione di Neri, della paura che una finitezza, professionale o teorica, scatenavano in lui e del conseguente ruolo dell'«eterno debuttante» interpretato dal filosofo ${ }^{39}$. V'è nondimeno una decisione che assume i connotati della positività, dell'apertura, una decisione che non comporta una cesura - etimologia da Neri spesso evocata nei dialoghi con gli amici ${ }^{40}$-: quella dell'«azione giusta» ${ }^{41}$, che non preclude la via al pensiero, ma lo crea e invita a ripensare e a recuperare dalla banalità le cose del mondo. L'«azione giusta» sta quindi anche in quel, a prima vista piano e, per lui, consueto atto dell'entrare in chiesa, metafora dell'accettazione coraggiosa del 'non finito', del non concluso. Nella, forse voluta, teatralità della prosa del quadernetto, sta tutto il valore di un gesto che, lungi dal saturare il senso del mondo imbrigliandolo in rigidi schemi, si lascia alle spalle le prevaricanti e opprimenti epifanie dello scontato e dell'imposto, per seguire una strada che non conduce ad approdi definitivi e confortanti, ma dischiude al pensiero la possibilità di un'alternativa, di un nuovo inizio; così, dal fianco destro di S. Pietro in Gessate, protetto da una cancellata, Neri compie, ancora una volta, la sua scelta: «Di lì guardo fuori, il P‘alazzo» di Giustizia〉 è più lontano, non incombe più. Decido di entrare».

\footnotetext{
${ }^{39}$ Cfr. A. Vigorelli, "Il filosofo come eterno debuttante", Materiali di Estetica. Terza serie, III/1, 2016, pp. 134-136.

${ }^{40}$ Cfr. L. Marelli, "Un filosofo indecisionista a Milano", cit., p. 30.

${ }^{41}$ Ivi, p. 31.
} 


\author{
Fondo Guido Davide Neri* \\ Serie 1: Quaderni \\ Cartella 9 \\ unità archivistica 98: Inverno 1992, 1992
}

\author{
$|54 \mathrm{r}|$ \\ $>$ S. Eustorgio $<\underline{\mathrm{S}\langle a n>\mathrm{P}\langle\text { ietro in Gessate: }}$ \\ si difende bene con l'aiuto \\ di qualche pianta \\ mettendosele intorno \\ e piazzetta a ciotoli
}

\author{
ore 17.45 \\ popolo al riparo fuori da \\ S‘an〉 Piietro〉 in Gressate` \\ fotografare fianchi $\rightarrow$ largo liceo \\ $\rightarrow$ via ... \\ e panchine con gente \\ $|54 \mathrm{v}|^{* *}$
}

Inutile pensare a come doveva stare in origine, quando aveva spazio intorno e non la sovrastavano blocchi quadrati dai due lati. Invece constato che, asserragliata, si difende. Dispone di una piazzetta minima, ma con i suoi ciotoli e 2 file di cinque alberi per parte. Grosse panchine di granito sotto gli alberi su cui trovare riparo come su un'isola della gente vecchia e stanca, che si immagina espulsa dal traffico antistante, dallo stesso andirivieni frettoloso del marciapiedi antistante.

(Due blocchi di pietra e due bancarelle di libri >ribadiscono<)

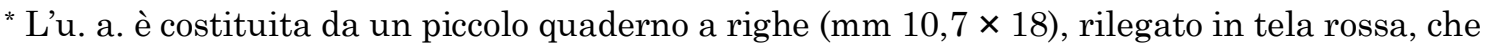
apprendiamo dalla controguardia posteriore essere stato impresso a «Shanghai. China». Sul piatto anteriore è stampato in oro "Diary», mentre sullo stesso e al dorso Neri ha aggiunto a pennarello nero, rispettivamente, «INV. 1992» e "INVERNO 1992». Le cc. sono numerate, a penna blu, 1-78, a partire dalla pagina di sguardia sino alla controguardia posteriore. La trascrizione è stata condotta secondo criterî conservativi: sono state pertanto rispettate la punteggiatura e l'ortografia originali. Le parentesi uncinate rovesciate $><$ racchiudono porzioni di testo cassate nell'autografo, mentre le parentesi angolate $\diamond$ integrano abbreviazioni o ricostruiscono per congettura segmenti di testo lacunosi. Non si riportano tutte le lezioni cassate, ma solo quelle significative da un punto di vista sostanziale. Le parentesi tonde e quadre sono da ricondurre all'autore, le graffe $\{$ ad interventi del curatore. ** A cavaliere delle cc. num. $54 v$ e $55 r$, Neri ha scritto, sul margine alto della pagina del quaderno: "Otazk: si può assumere questo status della chiesa come edificio quā prova [ontologica] della consistenza del pensiero che la sottende?».
} 
Si difende, intendevo, con mezzi spirituali. Girandole intorno da via Corridoni (il retro) in via ... mi tiene aderente lo sguardo, attaccato al mattone scuro delle sue cappelle laterali come se $\mid 55 \mathrm{r}$ | volesse difendere me, darmi almeno l'idea che c'è pure qua〉lcosa d'altro e che non tutto si esaurisce nella rabbia del traffico, nei tratti duri e cattivi dei palazzi. Approfitto dell'ora in cui si apre il cancello del liceo serale?, per percorrere l'altro fianco $>$ protetto<. Qui c'è più spazio e più quiete per osservare il corpo basso e scuro, ma ben modellato, e la sagrestia rinascimentale (?) che però non contrasta. Il "valore di antico" legato al mattone fuligginoso non guasta e mi domando con una certa apprensione se una pulitura troppo chiara non rovinerebbe l'effetto. Di lì guardo fuori, il P〈alazzo〉 di Giiustizia〉 è più lontano, non incombe più. Decido di entrare.

Entrare in una chiesa non è come entrare in cinema \{cinema sostituisce >un ufficio o in un bar $<$ \}. Dove sto entrando, intanto? A casa mia o in una casa d'altri? Il fatto che la $|55 \mathrm{v}|$ porta sia aperta mi invita a considerarmi un po' a casa mia; anche le moschee che ho visitato in Algeria e in Turchia mi accoglievano benigne benché fossi un infedele. Qui le cose sono diverse perché tra me e queste chiese c'è una storia più drammatica, la mia storia personale, la mia rottura con la religione della mia famiglia. Con quale "statuto" mi riaffaccio qui dentro?

Per essere più vicino al vero devo dire che è solo in un senso ben definito che io mi pongo questo problema, perché in realtà io entro e esco assai spesso nelle chiese - e non solo quando devo assistere a dei funerali. Proprio quando, verso i 12 o i 13 anni, ho cominciato a abbandonare | 56r | le chiese come luoghi di culto, mi è nata una vera passione per l'arte della n〈o`sıtra〉 tradizione e ho cominciato a frequentarle come musei e opere di architettura. $>$ Ho studiato $<$ Per diversi anni mi sono lasciato consumare da questa passione, interpretandola come passione del tutto "laica". Mi favoriva l'estetica visibilistica: che dappertutto si affacciassero Madonne e Santi, Annunciazioni), Crocifissi, Res‘urrezioni e Ascensioni, mi era indifferente; e del resto si vedono le stesse cose anche a Brera o agli Uffizi. Mi stupiva piuttosto un amico americano, forse ${ }^{* *}$, che lamentava un "troppo pieno" di madonne a B., o un amico ebreo piccolo collezionista, che non anche lui \{sic\} amava le madonne medievali. La mia formazione visibilistica mi diceva che non dovevo minimamente accorgermi della natura del soggetto: che il "soggetto vero" era comunque un altro. Naturalmente c'era del vero, eppure non era così semplice. Un altro $|56 \mathrm{v}|$ modo di rientrare in chiesa, più recente, è stato con la musica. Adoro la musica ma ne ho meno esperienza. La notte di Natale di ... anni fa, con degli amici, a S. Marco, poi a S. Ambrogio: a S. Marco la ... di H«äındel (?) Anche qui fu la musica "pura"? In Chiesa come al Conservatorio? Hı̈̈ındel come ...? Natale come Capodanno? In verità non era così. Eravamo nel periodo in cui stava cambiando un'epoca: si usciva da una guerra, da una guerra civile e insieme di generazioni. Non importa se mi ero tenuto piuttosto da parte in questa guerra. L'amico che era con me aveva un famigliare in carcere. Nel giro carcerario lui, col quale avevo commentato quasi

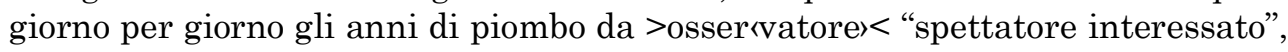
aveva avvicinato singolari figure di cattolici, che in veste di ... avevano partecipato attivamente alla pacificazione. La chiesa $|57 \mathrm{r}|$ con cui avevamo litigato, io e forse anche lui, non era più la stessa? Lo sapevamo, ma ci sono voluti quegli anni per farne l'esperienza. Per conto mio, avevo vissuto con meraviglia e con ammirazione le vicende di Solidarnosc, visti gli operai inginocchiati in pubblico, dove un prete diceva la messa. Accettato con animo aperto l'incredibile

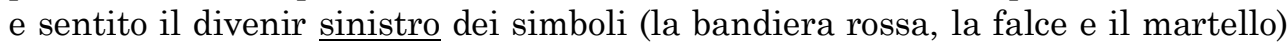

Materiali di Estetica - N. 8.2: 2021, Pagina 488 
che mi aveva catturato in gioventù. La musica di $\mathrm{H} \ll \ddot{a})$ ndel in S. Marco non era "pura forma sensibile".

Cos'era allora? Mi stavo convertendo? Rinnegavo tutto il mio passato e mi apprestavo a giocare il ruolo del figliol prodigo? Qualche amico, anche molto vicino, lo ha sospettato. Ma se è vero che la rottura con la religione di famiglia era e restava un trauma (comportava la perdita di contatto con tanti parenti cari, che non mi potevano capivano \{sic\}, - così almeno pensavo - così come la $|57 \mathrm{v}|$ scelta politica di sinistra la determinava con altri) io non avevo molto da rimproverarmi. Non con leggerezza avevo tagliato i ponti. Questa chiesa in quegli anni, quando cominciavo a pensare e volevo sapere, mi aveva risposto solo con catechismi, con le superstizioni, preti supponenti e irosi anche al liceo. Processioni per la Vergine e contro l'anticristo, come per invocare la pioggia. Amministrazione forzata dei sacramenti. Anche senza avere esperienza diretta del peggio che la chiesa era stata e che era tuttora, ho fatto l'esperienza personale della chiesa come sepolcro imbiancato, "casa dei morti" come la chiamava Arntonio〉 B «anfi〉, che stava per diventare il mio maestro di filosofia.

Così la filosofia mi aveva avuto tutto per sé. Il cammino che percorrevo |58r| non era molto originale, molti vi si riconosceranno. Kant per quel tanto che lo potevo indovinare prima ancora di studiarlo a scuola mi liberava dalla falsa teologia e con ciò anche dal catechismo. Feuerbach e Marx ribaltavano la frittata teologica in antropologia. Banfi, da raffinato neokantiano fattosi populista, completava l'opera ironizzando su quei suoi colleghi metafisici che avevano una linea diretta col Padreterno. Più tardi ho potuto conoscere meglio Kant e capire quanto fasullo fosse il carducciano "Decapitaro ..." Ho anche avvicinato singole figure e opere di filosofi "laici" e "cattolici" che cercavano di andare oltre. Un esempio? Ernst Bloch che viveva la Bibbia come cosa sua, e la leggeva con la stessa passione con cui leggeva Marx nella sua "corrente calda". Non che mi convincesse in tutto, con il suo umanismo un po' enfatico (e non solo "utopico"), $|58 \mathrm{v}|$ ma leggere Bloch era come ascoltare Hiäindel o Bach. Si toccava il profondo, non il chiaro e distinto. ("meglio un el Greco..."). E si capiva - anche dalle sue vicende con il comunismo reale - quarlcısa〉 di quel che al comunismo mancava. Bloch era quasi solo, da comunista, a affrontare così come il problema della religiiones, anche quello della morte. Una meditaz. approfondita su Heidegger venne poco dopo; e è ancora in corso. Ma come potrei non essere fortemente sensibile alla sua critica alla metafisica ont-teologica, come $>$ non $<$ potrei non sentire l'originalità nella problematica "fenomenologia" sull'Essere, che "non è un predicato reale" - e che non è Dio. Qui non è il luogo per una discussione $|59 r|$ teologica, in cui non avrei niente da insegnare a nessuno, solo quarlche riflessione e reazione personale. Ma era per dire che non rinuncio a un rigo della mia filosofias. Soltanto: non l'ho trovata nelle parti politiche cui mi ero avvicinato. Lì trovavo un nuovo catechismo, altrettanto borioso e gretto quanto quello da cui provenivo. Mi ero adattato, come tanti altri, a dissociare il discorso f́ilosoficor da quello politico-pratico, che poteva significare di più. Dopotutto $>$ gli uomini non< è l'essere degli uomini che conta, non la loro autocoscienza. Finché non dovetti convincermi che questa dissociazione riguardava me e i miei amici, ma non chi regnava in quel mondo. Il '68 di Praga e $|59 \mathrm{v}|$ poi la triste atmosfera da tardo impero mi convinse rapidamente che le cose erano a questo punto.

$>$ qqui continuare su Praga? $<$

Materiali di Estetica - N. 8.2: 2021, Pagina 489 


\section{Uno sguardo sensibile attraverso il reale}

di Paolo Karapedian paolo.karapedian@gmail.com

Ho conosciuto la figura di Guido Davide Neri attraverso il corso di Filosofia della Storia, tenuto dal prof. Amedeo Vigorelli nel secondo semestre dell'anno accademico 2019-2020, intitolato Eredità del secolo breve. Il corso, il cui obiettivo era quello di presentare il flusso storico novecentesco attraverso la lettura di Hobsbawm¹ ${ }^{1}$, aveva trovato nell'opera Aporie della realizzazione ${ }^{2}$ un supporto essenziale per orientarsi all'interno di quel grande esperimento metafisico, concretizzatosi nei territori orientali del Vecchio Continente, conosciuto con il nome di Socialismo Realizzato.

La lettura del volume di Neri, che poneva al centro della sua riflessione le esperienze di due generazioni di intellettuali della prima e della seconda metà del Novecento, contribuiva a stimolare in noi un certo interesse per i temi trattati e ci incuriosiva rispetto alla fisionomia del suo autore. Curiosità ulteriormente accresciuta dalla presenza, presso la Biblioteca di Filosofia dell'Università degli Studi di Milano, del Fondo Guido Davide Neri, fonte preziosa di informazioni e concreta possibilità di incontro col filosofo, con la realtà delle sue riflessioni teoriche e dei suoi scambi culturali. La notevole ricchezza che mi si dispiegava davanti, sia sul lato teorico che documentario, mi portò poi alla decisione di consacrare la mia tesi magistrale, alla figura di Neri.

Nello specifico il mio studio si è poi concretizzato in un primo tentativo di biografia intellettuale del fenomenologo milanese, il cui stile filosofico e di lavoro ben si è sposato con tale impostazione, costituendo pratica abituale per

\footnotetext{
1 E.J. Hobsbawm, Il secolo breve 1914-1991, BUR, Milano 2014.

2 G. D. Neri, Aporie della realizzazione. Filosofia e ideologia nel socialismo reale, Unicopli, Milano $2015^{2}$.
} 
Neri, fin dai primi lavori giovanili, il tornare ciclicamente su diverse tematiche affrontate, arricchendole di aspetti sempre nuovi.

Ho impostato la prima parte del lavoro, cercando di ricostruire il periodo e gli ambienti di formazione: il liceo, l'università, l'incontro con i tre Maestri: Antonio Banfi, Enzo Paci e Dino Formaggio, sottolineandone il ruolo decisivo per la genesi e l'evoluzione del pensiero neriano. Ho proseguito analizzando la prima produzione del filosofo milanese, come la curatela del saggio di Panofsky ${ }^{3}$, senza dimenticare di evidenziare il rilievo del periodo trascorso a Praga, durante il quale Neri ebbe modo di conoscere le dinamiche quotidiane di un paese del Blocco orientale e di stringere legami con intellettuali come Karel Kosík e Jan Patočka, il cui pensiero avrebbe incisivamente contribuito a far giungere in Italia 4 .

La seconda metà degli anni Sessanta vide pubblicato il primo libro teorico di Neri ${ }^{5}$ e la sua chiamata alla Penn State University, per un biennio di insegnamento; incarico che avrebbe assunto solo per un anno, decidendosi per il ritorno in Italia - chiamato da Formaggio a Verona - e iniziando così il suo trentennale impegno didattico nella città scaligera. Un periodo questo che coincise con una stagione importante della storia politica europea, nei confronti della quale Neri dimostrò, ancora una volta, di riservare particolare attenzione soprattutto nei suoi sviluppi oltrecortina: si vedano le analisi da lui compiute del complesso e sfortunato esperimento della Primavera di Praga.

La Cecoslovacchia non fu però la sola meta dei suoi viaggi: nel 1971, tra i primissimi italiani dopo la rivoluzione, andò in Cina, traendo ulteriori motivi per dubitare delle esaltazioni acritiche del Socialismo reale; avrebbe anche avuto modo di visitare la Germania Orientale, l’Ungheria e la Polonia, oltre

\footnotetext{
${ }^{3}$ E. Panofsky, La prospettiva come "forma simbolica" e altri scritti, a cura di G. D. Neri, con una nota di M. Dalai, Feltrinelli, Milano 1961.

${ }^{4}$ Su questo si può vedere L. Fausti, Guido Davide Neri tra scepsi e storia, Unicopli, Milano 2010, pp. 27-32.

${ }^{5}$ G. D. Neri, Prassi e conoscenza, Feltrinelli, Milano 1966.
} 
che di studiarne il pensiero di importanti intellettuali come Ernst Bloch, György Lukács e Leszek Kołakowski, al centro di una decisiva rivalutazione, insieme a Karel Kosík, che sfocerà in Aporie della realizzazione (1980). Il nuovo confronto con Banfi6, la riscoperta di Merleau-Ponty, l'approfondimento di Hannah Arendt avrebbero poi completato il quadro del lavoro di Neri negli anni Ottanta-Novanta.

La sagoma del Fondo è visibile in controluce dietro tutta l'impalcatura del mio lavoro di tesi; fin da subito ho compreso la sua imprescindibilità per l'accostamento a una figura tanto complessa e la sua capacità di restituirmi immediatamente l'umanità di Neri, grazie anche alla sola osservazione dei documenti biografici e dei taccuini ${ }^{7}$, spesso abbondantemente decorati di disegni e colori. Un quadro già stratificato a cui si deve aggiungere l'esistenza di due nuclei di volumi provenienti dalla biblioteca personale di Neri: uno custodito dalla Biblioteca di Filosofia e l'altro dalla Fondazione Feltrinelli. I libri, in molti casi contraddistinti da numerose sottolineature e da fitte annotazioni, risalenti anche a periodi diversi, danno l'immagine di studioso profondo e rigoroso, che torna sui suoi passi e rimedita costantemente il testo, senza darlo come acquisito una volta per sempre.

I Quaderni che più mi hanno affascinato sono quelli riguardanti il periodo americano ${ }^{9}$ e il viaggio cinese ${ }^{10}$, per la dimensione e la ricchezza di contenuti, ma anche per la rilevanza storiografica.

\footnotetext{
${ }^{6}$ G. D. Neri, Crisi e costruzione della storia. Sviluppi del pensiero di A. Banfi, Libreria Editrice Universitaria, Verona 1984.

${ }^{7}$ Cfr. in part. Fondo Guido Davide Neri, Serie 4, Sottoserie 1, Cartella 30, u. a. 1-58; Serie 1, Cartella 1, u. a. 1-15.

8 Il primo nucleo, conservato presso l'Università Statale di Milano, è costituito dal materiale librario/editoriale che Gabriele Neri, figlio del filosofo, ha donato nel 2009 alla Biblioteca di Filosofia: si tratta dei testi più esplicitamente filosofici in possesso di Neri. Alcuni libri erano però in precedenza confluiti presso la Fondazione Feltrinelli. Rispetto alla prima, questa seconda collezione consta di un numero di pezzi ridotto e ha carattere più marcatamente settoriale; si tratta infatti di opere di argomento storico-politico, soprattutto sulla realizzazione del socialismo in Cecoslovacchia.

${ }^{9}$ Cfr. Fondo Guido Davide Neri, Serie 1, Cartella 1, u. a. 15.

${ }^{10}$ Cfr. Fondo Guido Davide Neri, Serie 1, Cartella 2, u. a. 19-20.
} 
Il primo fascicolo si può inserire tra il 1967 e i mesi iniziali del 1968, periodo durante il quale Neri insegnò alla Penn State University, avendo modo di approfondire, oltre i temi oggetto del proprio lavoro, le dinamiche interne alla società americana e nello specifico universitaria, contrassegnata in quel momento dalla lotta degli studenti contro la Guerra in Vietnam e da numerosi esperimenti di controcultura, i cui esiti avrebbero influenzato i movimenti del '68 europeo.

La notevole mole del materiale conservato nel Fondo è prova tangibile della ricchezza di questa esperienza, le cui scelte culturali potremmo definire quantomeno coraggiose, impegnato come fu Neri a introdurre nel contesto statunitense autori come il croato Gajo Petrovič, incontrato durante il congresso Dialectics of liberation (Londra 15-30 Luglio 1967)11. Di Petrovič Neri, come testimonia una serie di appunti dattilografati in lingua inglese, recuperò, a partire dal libro che l'autore jugoslavo gli aveva dedicato ${ }^{12}$, le riflessioni sui problemi posti dalle prime opere di Marx, rintracciandone le critiche all'economicismo da una certa filosofia della prassi, i cui motivi presentavano dei punti di contatto con il pensiero di Kosík.

Nell'inverno del 1967 tenne una conferenza per gli SDS di State College, The different phases of the evolution toward socialism, il cui testo di undici pagine dattilografate in lingua inglese, ricco di disegni, è conservato. In esso Neri rifletteva sulle concezioni di comunismo e socialismo presenti in Petrovič ${ }^{13}$, del quale ricordava anche l'impegno nella rivista «Praxis» e nella scuola estiva di Korčula, su posizioni nei confronti dell'esperimento socialista jugoslavo che incontravano difficoltà di espressione e pubblicazione. Il ruolo specifico degli intellettuali nei territori del Blocco orientale, tra proselitismo

${ }^{11}$ Cfr. L. Fausti, Guido Davide Neri tra scepsi e storia, cit., p. 39.

${ }^{12}$ G. Petrovič, Marx in the Mid-Twentieth Century: a Yougoslav philosopher considers Karl Marx writings, INC, Garden City 1967.

${ }^{13}$ Cfr. G. Fusi, "Un filo rosso tra Italia e Boemia", Materiali di Estetica. Terza serie, III/1, 2016, p. 71. 
marxista e libertà espressiva, è del resto un tema centrale di questa conferenza, come lo sarà di molta produzione neriana.

Nella primavera dell'anno successivo Neri tenne un corso su Marx, la cui versione scritta, pervenutaci nella forma di un ampio dattiloscritto in inglese, costituisce la testimonianza più importante del periodo americano ${ }^{14}$. Esso si trova in una condizione, materiale e formale, tale da poter far pensare, dopo un lavoro accurato, a un suo totale recupero e a una possibile pubblicazione, visto anche il valore storico-culturale del documento, in cui Neri, dopo aver avuto modo di presentare a tutto tondo la figura del filosofo tedesco, propose all'uditorio diverse puntate sulla riflessione di autori contemporanei come Petrovič, Kosík e Marcuse.

L'altro fascicolo, sul viaggio cinese del '71, è di pochi anni successivo e presenta pagine molto suggestive. Neri, prima di partire, aveva compiuto diversi studi sulla Cina e la Rivoluzione Culturale, culminati in un articolo scritto a due mani con P. Gambazzi, in cui venivano analizzati i grandi cambiamenti, tra le classi e nella società più in generale, che stavano scuotendo il gigante asiatico ${ }^{15}$. I fogli conservati sono sia un diario di viaggio, con gli orari degli spostamenti, sia un laboratorio di impressioni, appunti, disegni e anche promemoria per studi futuri. Durante la visita Neri si attrezzò persino di un piccolo dizionario cinese nel tentativo, come aveva fatto durante il periodo praghese dei primi anni Sessanta, di avvicinarsi in qualche modo alla popolazione. Cosa che puntualmente fece.

Si vedano, a proposito, le carte dedicate alla visita a "Daqai», in cui Neri parla della condizione scolastica cinese, osservata grazie alla mediazione dei maestri operanti in quel distretto e all'analisi dei libri scolastici adottati. Questi ultimi, come vettore fondamentale della trasformazione culturale, risultavano strutturati secondo precise esigenze e finalità, che Neri riassume

\footnotetext{
${ }_{14}$ Cfr. Fondo Guido Davide Neri, Serie 1, Cartella 1, u. a. 15.

15 Cfr. G. Neri e P. Gambazzi, "La rivoluzione della Rivoluzione culturale proletaria", Compagni, I, 1970.
} 
in cinque punti: lotta di classe, servizio al popolo, amore per il lavoro (manuale), ideologia maoista sulla guerra popolare e formazione dei Continuatori Popolari della Causa Comunista. Altra caratteristica di questi testi da Neri sottolineata, è il loro carattere sperimentale16: se i libri precedenti alla Rivoluzione Culturale erano il prodotto di figure specifiche (intellettuali, professionisti ecc.), apertamente criticate e condannate entro la nuova direzione ideologica, ora essi sono il frutto dell'operato delle masse popolari; cosa che porta il filosofo a chiedersi quali siano i rapporti che sussistono, nell'ambiente cinese, tra gli artisti, le professioni e i posti di comando.

Lo scambio di informazioni coi locali non si riduceva poi alle sole dinamiche politiche e sociali, o alla quotidianità dell'esistenza in una terra così lontana, geograficamente e culturalmente, dall'occidente. Temi più marcatamente filosofici e linguistici emergono dalle sue annotazioni: è il caso dello scambio di battute sulla distinzione terminologica tra «Sixiang» (pensiero), e "Libun» (teoria). Per entrambi gli interpreti, Kao e Sun, le due espressioni sono chiaramente distinguibili, ma se, secondo Kao, la differenza è prettamente pratica, per Sun lo «Sixiang» indica qualcosa di più rispetto a «Libun», e il motivo risiede nel fatto che il primo vocabolo è una commistione di teoria e pratica, cosa che Neri riferisce istintivamente a un suo referente culturale: Franz Schurmann.

La presenza proficua di tali scambi non deve far dimenticare, d'altro canto, la presenza di una costante dell'atteggiamento di Neri verso le incarnazioni opprimenti del socialismo realizzato: la mal sopportazione nei riguardi del clima di attenzione e di controllo creato attorno al gruppo dei visitatori, da parte degli interpreti e dei funzionari di partito, con i quali Neri rischiò, con innocente irriverenza, di entrare qualche volta in contrasto ${ }^{17}$.

${ }_{16}$ Cfr. Fondo Guido Davide Neri, Serie 1, Cartella 2, u. a. 19.

${ }^{17}$ Cfr. L. Nahon, "Guido D. Neri ovvero lo studio della bonarietà", Materiali di Estetica. Terza serie, III/1, 2016, p. 107. 
Ci si è brevemente soffermati su questi due nuclei di documenti, nel tentativo di mostrare, presi due soli esempi, quale sia la ricchezza, ancora poco esplorata, che caratterizza il Fondo Guido Davide Neri, e come sia possibile, attraverso questo, giungere a lui attraverso un percorso privilegiato e far arrivare a noi, sempre di nuovo, la sua voce. 


\title{
«Nella rete della storia».'L'importanza dell'interrogazione sul senso della storia di Jan Patočka nel pensiero di Guido Davide Neri
}

\author{
di Sofia Elena Merli \\ sofiaelena.merli@gmail.com
}

In queste pagine mi propongo di dare voce ad alcune riflessioni di Guido Davide Neri sul rapporto tra fenomenologia e storia, così come emergono in vari appunti e scritti preparatorî conservati nel fondo archivistico conservato presso la Biblioteca di Filosofia dell'Università degli Studi di Milano ${ }^{1}$. La testimonianza e l'impegno filosofico di Neri appare, ancora oggi, fondamentale per aver radicato in Italia l'interesse rivolto a J. Patočka e al suo allievo K. Kosík, dando vita ad un originale asse di dialogo tra la fenomenologia post-husserliana e lo storicismo marxista ${ }^{2}$. Questo filo

\footnotetext{
* "Nella rete della storia» è il titolo di un'opera che Guido D. Neri intendeva dedicare all'indagine del rapporto tra fenomenologia e storia, ripercorrendo quella che è stata la sua personale esperienza a partire dagli anni Cinquanta, quando la fenomenologia si è trovata a confrontarsi con il marxismo-leninismo. Lavoro che non ebbe modo di portare a termine, ma che permette di far emergere alcuni aspetti centrali della sua ricerca ed impostazione filosofica. Cfr. F. Tava, Il rischio della libertà, Mimesis, Milano 2014, p. 169.

${ }^{1}$ Cfr. Fondo Guido Davide Neri, Serie 1, Cartella 12, u.a. 148 e Serie 3, Cartella 5, u.a. 39, «Nella rete della storia». Entrambi i documenti, il primo in forma manoscritta in un quaderno, il secondo in forma dattiloscritta, riportano il medesimo titolo «Nella rete della storia». Sul retro della seconda pagina del quaderno, inoltre, sono riportati a penna rossa quelle che evidentemente avrebbero potuto essere altre proposte per il titolo dello scritto: "Nelle reti della storia», "La fenomenologia presa nella storia», "La fenomenologia alle prese con la storia (contemporanea) vissuta (col presente)». In assenza di indicazioni dirette, si avanza un'ipotesi di datazione sulla base di alcuni elementi interni. In virtù della presenza di due citazioni intratestuali a p. 10 e p. 11 del dattiloscritto u.a. 39, che fanno riferimento rispettivamente alle pp. 103 e 1 del numero CCXXXII-CCXXXIII di «aut aut», possiamo assumere l'ottobre del 1989 come terminus post quem. Inoltre, grazie alla ricostruzione della cronologia dei programmi dei corsi universitari tenuti da G. D. Neri (cfr. L. Fausti, Guido Davide Neri tra scepsi e storia. Un percorso filosofico, Unicopli, Milano 2010, pp. 151-185) è possibile notare una corrispondenza tra le tematiche del dattiloscritto e quelle degli insegnamenti degli anni tra il 1988 e il 1991. Alla luce di questi elementi, si potrebbe ragionevolmente concludere, anche se non con assoluta certezza, che il dattiloscritto [u.a. 39] «Nella rete della storia" sia stato scritto tra la fine del 1989 e il 1991.

${ }^{2}$ Cfr. A. Vigorelli, "A partire da Patočka: itinerario filosofico di Guido Davide Neri”, גeússein, IV, 2012, pp. 141-163.
} 
particolare che unisce Milano e Praga inizia ad intramarsi durante il lungo periodo di studio che Neri trascorre a Praga tra il 1961 e il 1962.

L’importanza del soggiorno nella città praghese si sintetizza nel dialogo che Neri instaura personalmente con Patočka e Kosík, all'epoca relegati ad un importante, benché silenzioso, lavoro di rinnovamento filosofico, presso la Biblioteca dell'Istituto di Filosofia dell'Accademia Cecoslovacca delle Scienze ${ }^{3}$. Ed è poi nella conferenza tenuta da Enzo Paci a Praga nel 1962, in presenza di Kosík e Patočka, di cui Neri si fece arguto promotore, che possiamo individuare il concreto sigillo di questa connessione4. Dalla conferenza emerge come Paci, rimanendo legato ad una visione più conciliativa tra marxismo ed esistenzialismo, intenda ricercare, non senza una certa violenza ermeneutica, le tracce di una visione comune tra Husserl e Marx, giungendo a indicare la presenza di un solido intreccio tra il lavoro vivente di Marx e il soggetto vivente di Husserl ${ }^{5}$. Al contrario, Neri, soprattutto grazie alla mediazione della Dialettica del Concreto di Kosík, si apre alla rivisitazione del metodo fenomenologico che in quegli anni era stata inaugurata da E. Fink, M. Merleau-Ponty e J. Patočka, divenendo così più critico nei confronti della filosofia della storia marxista. È proprio attraverso il percorso filosofico di Neri che si registrano gli esiti più proficui e consistenti di questo incontro tra Milano e Praga, in una fenomenologia non più concepita

\footnotetext{
${ }^{3}$ Per una ricostruzione della biografia storico-intellettuale di Guido Davide Neri, con accenni anche all'esperienza praghese, cfr. L. Fausti, Guido Davide Neri tra scepsi e storia. Un percorso filosofico, Unicopli, Milano 2010.

${ }^{4}$ La conferenza di E. Paci, "Il significato dell'uomo in Marx e Husserl", aut aut, XXXVIII, 1963, pp. 10-21. Nel suo intervento Paci vede una comunanza di pensiero tra Husserl e Marx nella volontà di dissipare le astrazioni diffuse della società contemporanea, riportando lo sguardo verso ciò cui si indirizza il compito del filosofo: l'uomo concreto, l'uomo preso nella sua concretezza storica. Interpretando il movimento dall'astratto al concreto, quindi da una comprensione astratta, feticizzata della realtà ad una dimensione più autentica, Paci individua una forte analogia con il percorso inaugurato dalla fenomenologia.

${ }^{5}$ Cfr. F. Tava, Il rischio della libertà, Mimesis, Milano 2012, pp. 173-175.
} 
come "astorica" visione essenziale, bensì presa consapevolmente «nella rete della storia»6.

Neri nota come la dimensione della storia non appartenesse alle origini della fenomenologia, ma ne costituisse una contraddizione rispetto alle premesse. Nelle pagine degli appunti dattiloscritti per le lezioni veronesi del maggio 1991, spiega come il XIX secolo, cominciato con «una robusta coscienza della storia come di una realtà unitaria e progressiva», fosse paradossalmente sfociato in «una crisi rovinosa che investiva la possibilità stessa della Storia e dava luogo ad uno scetticismo relativistico che tendeva a chiudere le diverse epoche, i diversi tipi culturali, dentro sfere chiuse e incomunicanti» ${ }^{7}$. In questo stato della crisi della coscienza storica Neri individua poi lo sfondo e la situazione in cui nasce la fenomenologia all'inizio del secolo:

Io credo che il movimento fenomenologico nel suo insieme abbia aperto delle nuove strade nella comprensione della storicità, capaci di superare le difficoltà e le aporie insite nel loro rapporto. Tuttavia, la Fenomenologia non vi è arrivata in un balzo, ma per delle deviazioni e correzioni di rotta - e proprio su questi problemi si sono avute le discussioni e le rotture tra Husserl e i suoi allievi ${ }^{8}$.

Husserl, infatti, nel suo manifesto filosofico, La filosofia come scienza rigorosa, aveva tracciato una netta distinzione tra la filosofia come scienza, caratterizzata da uno sguardo sovratemporale, e lo storicismo di Dilthey, che insieme alle filosofie della Weltanschauung, avevano preteso di ridurre la filosofia alla filosofia della storia, quale realtà storicamente determinata e situata. Ma «la storia reale si vendicò di questi propositi husserliani»» ${ }^{9}$, poiché le ideologie che si diffusero a partire dagli anni Trenta lo costrinsero a un isolamento sempre più soffocante, facendogli maturare una nuova sensibilità verso i problemi della modernità e del presente, «così si risolse ad affrontare

\footnotetext{
6 «La "astorica" fenomenologia fece il proprio battesimo del fuoco in un presente storico dei più inquietanti che vi siano mai stati». Così scrive Neri nel saggio preparatorio intitolato "Nella rete della storia" (Fondo Guido Davide Neri, cit.). Cfr. anche A. Vigorelli, "A partire da Patočka: itinerario filosofico di Guido Davide Neri", cit., pp. 146-147.

${ }^{7}$ Fondo Guido Davide Neri, Serie 3, Cartella 19, u.a. 19, «Fenomenologia e storia», 1991, p. 5.

8 Ivi, p. 6, corsivo mio.

${ }^{9}$ Fondo Guido Davide Neri, Serie 3, Cartella 5, u.a. 39, «Nella rete della storia», p. 1.
} 
la crisi di una presa di coscienza storicamente orientata, che assegnava alla filosofia una nuova portata pratica» ${ }^{10}$.

Questo gesto di Husserl appare a Neri come un estremo tentativo di far fronte ai pericoli del presente, comportando per la fenomenologia l'ingresso della dimensione storica nel suo universo di discorso. Nel dattiloscritto Nella rete della storia Neri constata, quindi, come sia stato proprio il confronto della fenomenologia con la grande escatologia del secolo, il marxismo-leninismo, e con la sua realizzazione effettiva, ad averla, in un certo senso, costretta a dover fare i conti con la storia, e il suo senso ${ }^{11}$. Alla luce di questo confronto, si pone di fronte alla fenomenologia una domanda cruciale, così formulata da Neri:

Più in generale, l'atteggiamento è in grado di garantire un accesso privilegiato al "significato filosofico" della storia, anche quando il fenomenologo vi partecipa in prima persona, oppure la storia è una dimensione solo pragmatica, dove il sapere filosofico non gode di alcuna prerogativa e dove la strumentazione fenomenologica non è in grado di applicarsi? ${ }^{12}$.

Senza dare una risposta diretta a tale quesito, Neri, nell'interrogarsi sulle possibilità dell'atteggiamento fenomenologico di aprirsi a un nuovo sguardo, evidenzia il riemergere, sulla scena drammatica e contraddittoria della metà del XX secolo, del problema del senso:

\begin{abstract}
Come è propriamente formulabile il "problema della storia", e si tratta di un problema che consente una risposta risolutiva? Il pullulare delle diversità riduceva gli eventi umani a semplici "fatti", a un insensato susseguirsi di onde in un oceano dove sembrava avvenire di tutto e dove in realtà non avviene mai niente? Oppure il solo "senso" riscontrabile nell'evoluzione storica consiste nell'avvolgimento progressivo del mondo intero nella gabbia di una razionalità tecnologica, che assorbirebbe integralmente in sé il significato della ragione "europea"? $?^{13}$.
\end{abstract}

\footnotetext{
${ }^{10}$ Ibidem.

${ }^{11}$ Ivi, p. 3. Neri scrive: «Il tema, in sé tutt'altro che nuovo, si lascia forse leggere con maggiore distacco ora che quell'epoca sembra chiudersi e le prospettive generali si trasformano radicalmente» - e aggiunge a lapis a margine - «in cui sorgono nuove domande sulla dimensione della storia, oppure lasciandoci in cerca di un nuovo senso della storia».

12 Ivi, p. 2.

${ }^{13}$ Ivi, p. 7.
} 
Secondo Neri, le risposte più originali e interessanti sono racchiuse nelle riflessioni di chi ebbe modo di vivere l'atmosfera del socialismo reale, dove «limpressione generale era che lì davvero "lui" - o in verità qualcosa di sconvolgente e di tragico - era già passato, che l'aspettativa messianica fosse già trascorsa e che si vivesse nell'atmosfera del "dopo"»14.

All'interno di questo quadro, Neri appare profondamente colpito dal pensiero di Patočka ${ }^{15}$, per il quale la riflessione sulla storia divenne tanto centrale da riorientarne profondamente il pensiero fenomenologico. Operazione che aveva trovato il suo perno nella tematizzazione del concetto husserliano di mondo della vita, chiave di volta del calarsi della fenomenologia nell'universo storico-politico e dell'andare oltre la sua originaria impostazione metodologica. Ł̀ proprio attraverso le riflessioni patočkiane sulla Lebenswelt, che Neri riesce a compiere un fondamentale passo in avanti in questa direzione: «in effetti, sarà solo dopo aver fatto personalmente i conti con l'esperienza storica del socialismo reale e aver decretato il fallimento della escatologia storica marxista (nel libro del 1980, Aporie della realizzazione), che Neri si sentirà pronto a far propria l'idea di una radicalizzazione del programma fenomenologico, messo alla prova dalla storia contemporanea» ${ }^{16}$.

Significativo, in tal senso, appare il saggio Il mondo del lavoro e della fatica $^{17}$, dove Neri, riprendendo l'articolazione dei movimenti dell'esistenza umana con cui Patočka aveva delineato le modalità di accesso dell'uomo al mondo, percepisce l'esigenza di quest'ultimo di porre il problema del mondo naturale sotto l'egida della storia. L'obiettivo patočkiano era stato quello di far fronte all'oggettivismo e di rendere ragione della paradossale situazione

\footnotetext{
${ }^{14}$ Ivi, p. 13.

${ }^{15}$ Cfr. G. D. Neri, Un fenomenologo nel socialismo reale, in Fenomenologia e scienze dell'uomo, Clesp, Milano 1979, pp. 45-52. E cfr. l'intervista a J. Patočka, "Sulla filosofia e sui filosofi", in J. Patočka, Saggi eretici sulla filosofia della storia, CSEO, Bologna 1981.

16 A. Vigorelli, "A partire da Patočka: itinerario filosofico di Guido Davide Neri”, cit., p. 156.

17 G. D. Neri, "Il mondo del lavoro e della fatica", ora in Il sensibile, la storia, l'arte. Scritti 1957-2000, Ombre corte, Verona 2003, pp. 156-164.
} 
per cui il mondo moderno sembrava voler cancellare ogni traccia dell'esistenza naturale, ma allo stesso tempo appariva soccombere ad un altro tipo di "naturalità", legata alla perpetuazione del ciclo di mera riproduzione della vita. Patočka aveva maturato, quindi, un'idea di mondo naturale non in senso astrattamente soggettivistico, bensì quale mondo della prassi umana, della totalità, e luogo della vita concreta ${ }^{18}$, così da far fronte alle deformazioni messe in atto dalla tecno-scienza.

Con questo progetto Neri coglie in Patočka la volontà di restituire al mondo la sua complessità e problematicità, contro la riduzione all'oggettivazione e all'ovvietà. Nel riconoscergli questi caratteri Neri dà slancio all'aprirsi all'indeterminatezza della risposta sul senso e sigla il venire meno di soluzioni confortanti al problema e l'impossibilità della sua chiusura in una realtà definita: in quanto storia essa rimane e deve rimanere un problema, una apertura sempre di nuovo interrogante.

Anche solo in questi brevi schizzi, è possibile riconoscere in Neri un finissimo lettore del filosofo ceco, al quale riconosceva una posizione di primo piano nella tradizione fenomenologica novecentesca, tanto da considerarne il pensiero, insieme a quello di Merleau-Ponty, l'incarnazione più criticamente avvertita ed elaborata della fenomenologia, e da erigerlo a un punto di riferimento essenziale anche per la sua analisi sul destino dell'Europa ${ }^{19}$. Non a caso aveva progettato di concludere il corso universitario su La percezione della storia. Unità e pluralità dei mondi storici e Filosofia della storia del Novecento e del presente, nel duplice segno di Patočka e Merleau-Ponty; la breve malattia e la prematura morte non gliene dettero il tempo. Nei suoi appunti si ritrova dispiegato l'urgente e scottante tema della residua possibilità per la storia di avere ancora un senso. L'inquietudine che si avverte

\footnotetext{
18 J. Patočka, Il mondo naturale e la fenomenologia, a cura di A. Pantano, con uno scritto di G. D. Neri, Mimesis, Milano 2003, pp. 143-145. Raccolta a cui Neri stava lavorando nell'ultimo periodo della sua vita e che non fece in tempo a vedere pubblicata.

${ }^{19}$ Cfr. G. D. Neri, "L'Europa dal fondo del suo declino", ora in Il sensibile, la storia, l'arte, cit., pp. 266-291.
} 
in Neri fa risuonare le connessioni tra i due autori, associando alla visione patočkiana del senso della storia come capacità di assumerne la problematicità, la risposta di Merleau-Ponty sulla sua possibilità, «ma non senza un essere come l'uomo che è capace di raccoglierlo» ${ }^{20}$. In queste parole il filosofo milanese racchiude quel percorso e quella tradizione teoretica animata dalla costante tensione tra fenomenologia e storia, della quale è stato forse l'erede più significativo ${ }^{21}$.

${ }^{20}$ Fondo Guido Davide Neri, Serie 3, Cartella 18, u.a. 38, «Percezione della storia. Unità e pluralità dei mondi storici», p. 28.

${ }^{21}$ Cfr. A. Vigorelli, “A partire da Patočka: itinerario filosofico di Guido Davide Neri”, cit., p. 163. 